\title{
Standardizing MELD Exceptions: Current Challenges and Future Directions
}

\author{
David S. Goldberg • Kim M. Olthoff
}

Published online: 6 September 2014

(C) Springer International Publishing AG 2014

\begin{abstract}
Since Model for End-Stage Liver Disease (MELD)-based allocation was implemented in 2002, a system of exception points has been in place in order to award increased waitlist priority to those patients whose severity of illness or risk of complications are not captured by the MELD score. These exceptions, categorized as standardized and nonstandardized, have been used with increasing frequency over time. Several challenges to the exception point system have emerged, including lack of standardization in the criteria used to approve such exceptions, geographic variability in the use and approval of such exceptions, and a limited evidence base to support certain exceptions. Herein, we summarize the current implementation of exception points, the challenges facing
\end{abstract}

\section{S. Goldberg}

Division of Gastroenterology, Department of Medicine, Perelman School of Medicine, University of Pennsylvania, Blockley Hall, 423 Guardian Drive, Room 703, Philadelphia, PA 19104, USA

\section{S. Goldberg}

Center for Clinical Epidemiology and Biostatistics, Perelman School of Medicine at the University of Pennsylvania, Philadelphia, PA, USA

\section{S. Goldberg $(\square)$}

Leonard Davis Institute of Health Economics, University of Pennsylvania, Blockley Hall, 423 Guardian Drive, Room 703, Philadelphia, PA 19104, USA

e-mail: david.goldberg@uphs.upenn.edu

\section{K. M. Olthoff}

Division of Transplant Surgery, Department of Surgery, Perelman School of Medicine, University of Pennsylvania, Philadelphia, PA, USA

e-mail: kim.olthoff@uphs.upenn.edu

\section{K. M. Olthoff}

Penn Transplant Institute, Hospital of the University of Pennsylvania, 3400 Spruce Street, 2 Dulles, Philadelphia, PA 19104, USA the transplant community, and suggestions for improving and standardizing the current exception point system.

Keywords MELD exceptions · Standardized exceptions · Regional review boards

\section{Introduction}

Since February 27, 2002, allocation of livers to waitlisted transplant candidates has been based on an urgency-based disease severity model. Prioritization on the waitlist is determined by a patient's Model for End-Stage Liver Disease (MELD) score, a calculated risk score based on a patient's bilirubin, creatinine, and international normalized ratio (INR) and has been validated to accurately predict a patient's 3 month waitlist survival $[1,2]$. However, as is the case with any mathematical model or risk score, there is imperfect correlation between MELD score and waitlist outcomes. There are some patients that may be 'sicker' than their MELD score, due to multiple complications of portal hypertension, inaccurate measurements of renal function due to a low creatinine from low muscle mass, or have complications of liver disease requiring timely transplant that are not captured by the MELD score. Since implementation, a system of exception points has been in place in order to award increased waitlist priority to those patients whose severity of illness or risk of complications are not captured by the MELD score, but may have equivalent mortality risk to those with higher MELD scores.

Broadly speaking, MELD exceptions fall into two categories: (i) standardized exceptions, and (ii) non-standardized exceptions $[3 \bullet, 4 \bullet, 5]$. Standardized exceptions are those conditions for which there are sufficient data to warrant allocating automatic exception points to patients meeting formalized exception criteria (i.e., hepatocellular carcinoma $[\mathrm{HCC}]$ ). By 
contrast, non-standardized exceptions are those conditions which are deemed important by the transplant team but for which the risk of mortality is not as clear-cut, and thus require review on a case-by-case basis (i.e., cholangitis, refractory ascites, hyponatremia).

Within each United Network for Organ Sharing (UNOS) region, a regional review board (RRB) is charged with reviewing and approving applications for exceptions http:// optn.transplant.hrsa.gov/PoliciesandBylaws2/policies/pdfs/ policy_8.pdf. There are guidelines to help govern the composition and structure of each RRB, although each region has discretion as to how the RRB is set up. Each RRB is chaired by the Regional representative to the Liver/ Intestine committee, and while each active liver transplant program has the opportunity to be represented, the number of members of an RRB varies by region. As some regions comprise a very large number of centers, certain RRBs rotate membership. Each RRB is expected have representation from hepatology and surgery, and can also include non-transplant healthcare providers, and/or non-medical (public) representatives, although in practice this occurs infrequently.

Decisions on approval or denial of exceptions, and the policies for approving exceptions vary by conditions, and will be discussed below. For exceptions that do not receive automatic approval, and require a vote by the RRB, the application may be approved or denied, with centers having the option of appealing a denial, and/or resubmitting with fewer requested exception points http://optn.transplant.hrsa.gov/ PoliciesandBylaws2/policies/pdfs/policy_8.pdf.

Over time, there has been a continuous increase in the number of waitlist candidates with MELD exception points, for both standardized and non-standardized exceptions [6]. In fact, in recent years, over one-third of transplant recipients had received exception points, with nearly two-thirds having an exception for HCC within Milan criteria (Fig. 1) [6, 7]. In this article, we will review the current state of MELD exceptions, focusing on the most common indications for exception points, highlight the current challenges of the MELD exception system, and discuss future directions or improvements in the system.

\section{Standardized Hepatocellular Carcinoma (HCC) Exception Points}

The most common indication for MELD exception points is HCC. In the nascent years of liver transplantation, it was recognized that the number and size of lesions in a transplant recipient were strongly associated with post-transplant outcomes [8]. Specifically, the best post-transplant outcomes were seen in recipients with one tumor $\leq 5 \mathrm{~cm}$ in diameter, or 2-3 tumors, each $\leq 3 \mathrm{~cm}$ (Milan criteria) [8]. Prior to the implementation of MELD-based allocation, post-transplant outcomes in patients with HCC were suboptimal $[9,10]$.
Since 1998, patients with HCC within UNOS criteria have been eligible for upgraded priority, and since the inception of MELD-based allocation in 2002, Milan criteria became UNOS criteria, and waitlist candidates with $\mathrm{HCC}$ within criteria were awarded standardized exception points to increase waitlist priority, with the intended goal of expediting transplantation before tumor burden exceeded criteria, thus compromising post-transplant outcomes [11••]. Although such a policy partially contradicted the mortality-based MELD model, it was widely accepted by the transplant community. These standardized HCC exceptions receive automatic approval provided they meet all UNOS criteria, and do not require a formal review and/or vote by the RRB.

Initially, early MELD exception policies over-prioritized waitlist candidates with $\mathrm{HCC}$ by initially awarding them 29 points (February 2002-April 2003), and then decreased this to 24 points (April 2003-January 2005), with subsequent upgrades every 3 months. There was little drop-off from the waitlist and a revised mortality risk curve demonstrated that 22 points should initially be given, rather than 24 ; thus the policy was changed again in March 2005, with exceptions only awarded for T2 lesions $[12,13]$. However, several recent publications have demonstrated that even the current policy over-prioritizes HCC patients, with significantly higher transplantation rates with correspondingly lower waitlist mortality rates in comparison with the non-exception waitlist population [12-14].

The issue of lower waitlist mortality and higher transplant rates for HCC exception recipients is just one challenge with attempts to optimize and standardize HCC exceptions. All waitlist candidates with HCC within UNOS criteria are eligible for HCC MELD exceptions, yet the risk of tumor progression is not uniform in this group of patients. HCC exception point recipients with a single tumor that has a complete response to loco-regional therapy and a low alphafetoprotein has exceedingly low risks of waitlist dropout, suggesting that these patients should receive fewer exception points or upgrades at longer intervals [15]. Second, the full impact of the growing number of HCC exception patients on the broader waitlist population is unknown. With increasing numbers of patients receiving exception points, the mean MELD score needed to receive an organ offer also continued to increase. Another significant issue is that of geography. A total of 22 MELD exception points in 'low-MELD' regions such as region 3 may allow a patient with a favorable blood type to be transplanted within a few months of waitlisting, as compared with over a year in 'high-MELD' regions such as region 5, where drop-off for HCC is now approaching dropoff for other diagnoses [11••]. The issue of geographic disparity may pose the greatest stumbling block in determining the best course for awarding HCC MELD exceptions.

The UNOS Liver and Intestinal Organ Transplantation Committee has put out two proposed modifications to the 
Fig. 1 Use of exception points for deceased donor transplant recipients from February 27, 2002 to September 30, 2013 (based on OPTN/UNOS data as of January 20, 2014). $†$ Percentage reflects the proportion of transplant recipients with that specific type of exception at the time of transplantation. $H C C$

hepatocellular carcinoma, OPTN Organ Procurement and Transplantation Network, UNOS United Network for Organ Sharing

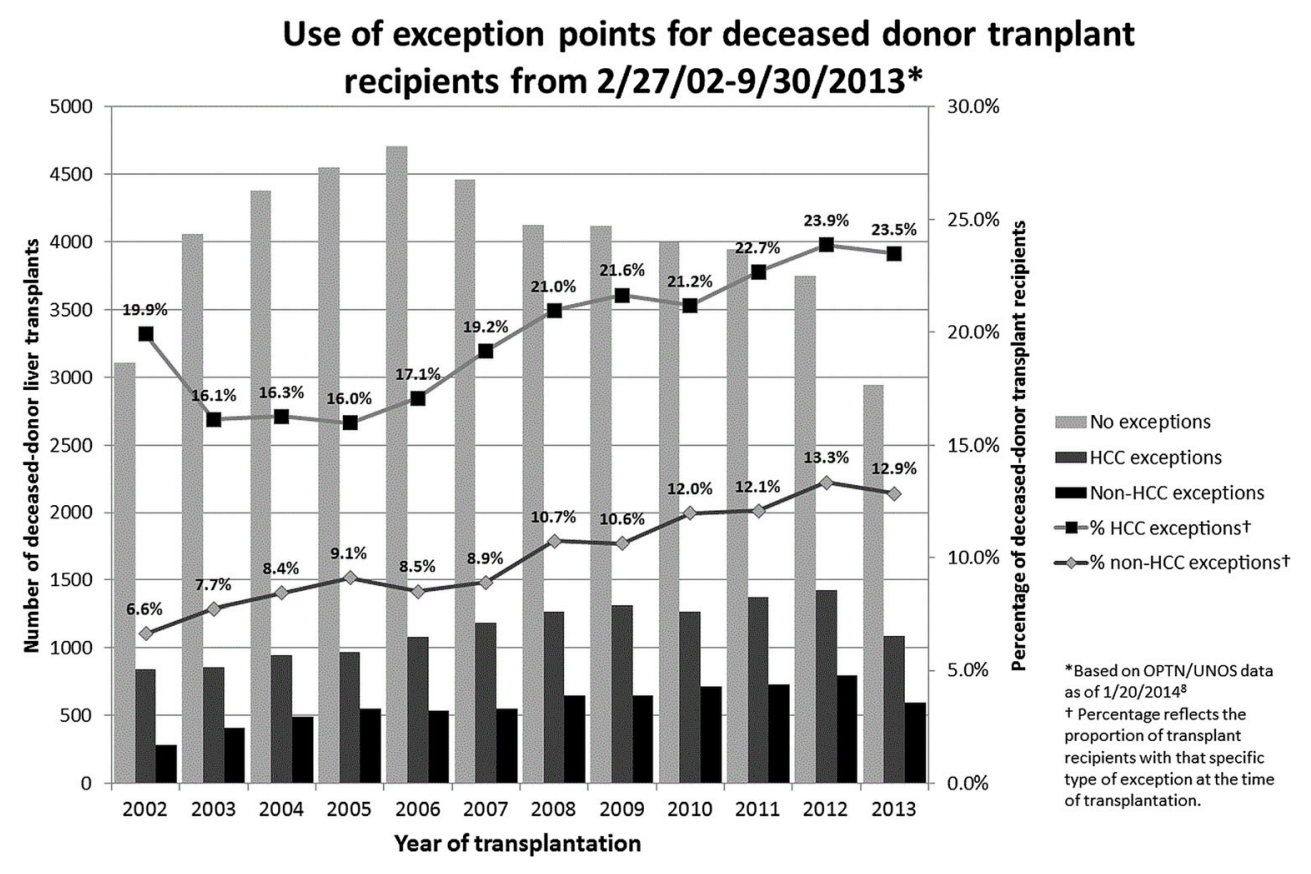

HCC MELD exception policy to try to address these concerns: (i) delaying assigning exception points to HCC patients within Milan criteria for 6 months after approval of the initial exception application; and (ii) capping the number of HCC MELD exception points at 34 [16]. However, standardizing HCC MELD exceptions remains a challenge, and there is no clear correct path forward. While there is rather broad support that HCC exceptions need to be revised, many transplant centers prefer to transplant these patients who are more clinically stable going into transplant and require fewer resources.

\section{Non-Standardized HCC Model for End-Stage Liver Disease (MELD) Exception Points}

In addition to standardized MELD exception points for $\mathrm{HCC}$, there is a separate group of patients with $\mathrm{HCC}$ eligible for exception points - those with tumors beyond Milan criteria who have undergone some type of 'down-sizing' intervention. There are no formal guidelines on awarding exception points for HCC beyond Milan criteria (either within or beyond University of California-San Francisco [UCSF] criteria [12, 17-19]). These cases all require formal review and voting by the RRB to determine whether the exception will be approved or denied.

While consensus recommendations have been published which suggest that exception points only be awarded to patients with HCC within UCSF criteria that have been 'downstaged' into UNOS criteria while demonstrating posttreatment stability, in practice, such guidelines are not followed. [14] In fact, since 2005, nearly $90 \%$ of exception applications for HCC within UCSF, and over $70 \%$ of exceptions for HCC beyond UCSF, were approved by RRBs, with nearly all of these approved exceptions not meeting consensus recommendations for criteria for exception points [14]. Despite a recent publication demonstrating similar post-transplant outcomes in select patients with tumors beyond Milan criteria, there are limited data to support this practice universally, and little transplant community support. Furthermore, these patients have a markedly higher risk of waitlist dropout [14]. Given the current organ shortage, and broader sharing of organs for critically ill patients, any consideration for expanding $\mathrm{HCC}$ exception criteria must take into consideration the potential downstream effects on the non-exception waitlist population.

\section{Other Standardized MELD Exceptions}

Other than HCC, there are several other conditions that are categorized as standardized MELD exceptions: hepatopulmonary syndrome (HPS), cholangiocarcinoma (CCA), familial amyloid polyneuropathy (FAP), cystic fibrosis, or portopulmonary hypertension (POPH). The standardized criteria have been vetted through the Liver/Intestine Committee and Board of Directors, and stipulate which patients should receive exception points provided they meet these criteria. Yet, RRBs currently have the authority to review the clinical scenario and award exceptions to patients who do not meet the specific clinical criteria. Specifically, when such exceptions are submitted, the RRB chair initially reviews the data provided in the clinical narrative, and if the patient meets the pre-specified UNOS criteria, the exception will receive automatic approval. However, if the chair determines that the case does not meet the specified criteria, the case is brought to the RRB for a formal vote. 
As an example, HPS MELD exception criteria are meant to be restricted to patients with a documented $\mathrm{PaO}_{2}<60 \mathrm{mmHg}$ on room air, in the setting of portal hypertension and a shunt, without evidence of underlying primary pulmonary disease [http:/optn.transplant.hrsa.gov/PoliciesandBylaws2/policies/ pdfs/policy_8.pdf]. A recent review of all national data on patients awarded HPS MELD exceptions since 2002 demonstrated that $10.8 \%(105 / 973)$ of such patients did not have a documented room-air $\mathrm{PaO}_{2}$ provided yet were awarded exception points, while an additional $13.4 \%$ (130/973) had a room-air $\mathrm{PaO}_{2}$ above the threshold of $60 \mathrm{mmHg}$ [20]. While such patients may in fact have had HPS, meeting Organ Procurement and Transplantation Network (OPTN) criteria for exception points, the data provided to UNOS did not document this. These deviations from current policy likely occurred because standardized criteria only dictate which patients automatically receive exception points, while RRBs have the discretion to also award exceptions to those not meeting such criteria. In response to such practices by RRBs, the UNOS Liver and Intestine Organ Transplantation Committee is revisiting policies for standardized MELD exceptions, and proposing to put into place more strict guidelines to dictate the practice of RRBs in awarding exception points for polycystic liver disease and neuroendocrine tumors.

\section{Non-Standardized MELD Exceptions}

While $\mathrm{HCC}$ is the most common indication for exception points, the second most common indication is for diagnoses categorized as 'other.' A subset of these 'other' exceptions is for HCC extensions, submitted as 'other' because standard HCC extension deadlines are missed. However, the bulk of these exceptions are non-standardized, and require a formal vote by the RRB, with all clinical data provided to the RRB from the transplant center in the form of an exception narrative. This broad category encompasses a broad range of conditions, including hyponatremia, refractory ascites or variceal bleeding, failure to thrive, and recurrent cholangitis, along with many other less common conditions for which no standardized criteria for awarding exceptions exists [4•, 21]. In fact, from May 1, 2012 through April 30, 2013, there were 920 requests for exception points for 'other' reasons, which represented $71.0 \%$ of all non-HCC exception requests during this time period [22]. Of these 920, 142 (15.4\%) are specifically for hyponatremia, while $42(4.5 \%)$ are for ascites, with greater numbers including these conditions among the 456 'other' applications without a specific diagnosis. While each of these conditions are associated with increased mortality $[23,24]$, there are no objective criteria in place for outlining who should receive such exceptions, and it varies significantly across regions. If there is implementation of the MELD-Na score currently being considered by the OPTN, this would obviate the need for exceptions given for low sodium or refractory ascites, as hyponatremia is seen as a surrogate for ascites in these patients.

Another group for which there are several 'other' exceptions is in patients with primary sclerosing cholangitis (PSC) and bacterial cholangitis. Nearly 300 patients with PSC and recurrent cholangitis received MELD exception points due to a concern for an increased risk of waitlist mortality [3•]. However, there are no data validating this concern, and the available evidence not only demonstrates that patients with PSC have a lower risk of waitlist mortality compared with the general waitlist population [25], but also that recurrent bouts of cholangitis do not confer any increased risk of waitlist dropout [26]. Non-standardized exceptions like this should only be given based upon evidence that those patients have a proven risk of increased waitlist mortality.

\section{Challenges in Standardizing MELD Exception Points}

Although the intended goal of implementing a system of MELD exception points was to equalize apparent inequities or limitations of the MELD score, there have been several challenges in standardizing the MELD exception policy. First, the number of MELD exception points awarded to those with approved standardized or non-standardized exceptions has not been evidencebased nor equated with their true risk of waitlist mortality. HCC exceptions are meant to award points that are associated with a $15 \%$ risk of waitlist mortality, with additional points added every 3 months that would be equivalent to a $10 \%$ additional risk of waitlist mortality. As has been demonstrated in several papers, waitlist candidates with MELD exceptions have a much lower risk of waitlist dropoff. [11••, 21, 27] The ideal mechanism to determine the necessary number of exception points would involve complex modeling based on current waitlist data. However, such attempts are hampered by the current MELD system that prioritizes patients to the point of very low waitlist dropout. Without sufficient outcomes, such modeling will ultimately be limited. Furthermore, a one-size-fits-all approach to awarding exception points does not account for the broad clinical spectrum of patients, even among similar diseases. A patient with a single 2-cm HCC that responds well to loco-regional therapy would likely have a much different risk of tumor progression than a patient with three tumors, each $2 \mathrm{~cm}$ in size, with persistent viability after two treatment cycles; [15] yet both would be awarded the same number of points as each are within Milan criteria.

Second, there is well recognized geographic variability in the MELD score at transplantation, ranging from the mid-30s for certain blood types in the most competitive donor service areas (DSAs), to the low 20s in others [28]. Thus, a policy that 
awards the same number of exception points to all patients, regardless of geographic considerations, results in inequitable access to transplantation. Median waiting times prior to transplantation for waitlist candidates with 22 HCC MELD exception points is $<90$ days in certain parts of the country, while it is $>1$ year in others [11••]. Standardizing MELD exceptions requires not just standardizing criteria for awarding such exceptions, but ensuring that being awarded such exceptions affords patients the same opportunity to be transplanted, regardless of geography.

Third, there are marked geographic differences in the number of non-standardized exception applications that are submitted, and the subsequent approval rates of such applications $[3 \cdot, 4 \cdot, 21]$. In fact, even within individual UNOS regions, there is significant variability at the center level in the submission, and subsequent approval of these non-standardized applications [4•]. There are several mechanisms to help explain these differences, but the root cause stems from a lack of standardization in the MELD submission and approval process. While the mean MELD at transplant may differ between regions, thus causing the amount of points awarded to differ, there should not be such variability in the standards that are used to award exception points. There needs to be attention focused on requiring more uniformity in the criteria used across all regions. Lastly, there is a lack of data for mortality risk or disease progression for some of these 'other' diagnoses.

\section{Conclusion}

Potential Suggestions to Standardize MELD Exceptions

Although the intention of the MELD exception system was to give increased priority to those patients with complications of liver disease not captured by the MELD score, there have been challenges to truly standardizing the process. Given this, we would propose several potential mechanisms for improving the policy.

1. Developing strict guidelines for non-standardized exceptions for RRBs. Such guidelines would clearly specify which conditions are eligible for such exceptions and the necessary criteria for awarding such points. RRB members would also be educated prior to their term, and instructed not to haphazardly award exception points to those patients who do not meet such criteria. RRB chairs should be held accountable for the decisions made by the RRB members if they do not utilize these guidelines in a uniform fashion.

2. For conditions for which standardized criteria exist (i.e., HCC within UNOS criteria, HPS with a $\mathrm{PaO}_{2}<$ $60 \mathrm{mmHg}$ ), exception points should be limited to those meeting these criteria. These criteria were developed based upon current data and outcomes. If new evidence evolves demonstrating that such criteria need to be revised, then policies can change. However, the HPS example, whereby many patients have oxygenation values above the pre-specified threshold, highlights the need for compliance with protocols.

3. When standardized criteria exist for awarding exception points, the necessary objective documentation is needed for diagnoses, and must be submitted prior to awarding exception points. For HCC, centers are required to document the size of tumors, and their radiographic appearance. Akin to this, HPS (and other conditions) exceptions should only be approved with the necessary data (i.e., room-air arterial blood gas, imaging study documentation, right-to-left shunting). One potential mechanism would be to program the applications for these exceptions into UNet, as is done for HCC.

4. Unless the method for prioritization of patients changes from the current urgency-based model, then exception points based on quality of life should not be permitted. This is too subjective a measure. The possible exception to this may be in the pediatric population where growth, development, and progress in school are important measures of the severity of liver disease.

5. Ideally, responsibility for reviewing and approving non-standardized exception point applications should fall to a national review board in order to assure uniformity of decision making across all regions [4•]. The current geographic variability in exception points leads to differential prioritization of patients with similar conditions, as a function of not only UNOS region, but transplant center. However, the logistics of such a national board are substantial, and include identifying who should comprise such a board, the volume of cases that would need to be reviewed, and how cases would be assigned. In addition, it is recognized that there are some local and regional nuances that cannot be completely appreciated by a national committee. Given these logistical and cultural barriers, at the very least, further efforts are needed to standardize guidelines for approval of exceptions across regions, and employ mechanisms to continually review and monitor RRBs for compliance with such guidelines.

\section{Compliance with Ethics Guidelines}

Conflict of Interest Dr. David S. Goldberg received a grant from Bayer HealthCare.

Dr. Kim M. Olthoff declares no potential conflicts of interest.

Human and Animal Rights and Informed Consent This article does not contain any studies with human or animal subjects performed by any of the authors. 


\section{References}

Papers of particular interest, published recently, have been highlighted as:

- Of importance

-. Of major importance

1. Kamath PS, Wiesner RH, Malinchoc M, et al. A model to predict survival in patients with end-stage liver disease. Hepatology. 2001;33(2):464-70.

2. Wiesner R, Edwards E, Freeman R, et al. Model for end-stage liver disease (MELD) and allocation of donor livers. Gastroenterology. 2003;124(1):91-6.

3. Goldberg D, Bittermann T, Makar G. Lack of standardization in exception points for patients with primary sclerosing cholangitis and bacterial cholangitis. Am J Transplant. 2012;12(6):16031609. This study explores the use of MELD exceptions for patients with PSC and bacterial cholangitis, and the lack of standardization of the review process.

4. Goldberg DS, Makar G, Bittermann T, Benjamin F. Center variation in the use of non-standardized MELD exception points. Liver Transpl. 2013. This study examines the variability in the use and approval of MELD exceptions between liver transplant centers within each UNOS region.

5. Freeman Jr RB, Gish RG, Harper A, et al. Model for end-stage liver disease (MELD) exception guidelines: results and recommendations from the MELD Exception Study Group and Conference (MESSAGE) for the approval of patients who need liver transplantation with diseases not considered by the standard MELD formula. Liver Transpl. 2006;12(12 Suppl 3):S128-36.

6. Thuluvath PJ, Guidinger MK, Fung JJ, Johnson LB, Rayhill SC, Pelletier SJ. Liver transplantation in the United States, 1999-2008. Am J Transplant. 2010;10(4 Pt 2):1003-19.

7. According to OPTN/UNOS data as of January 10.

8. Mazzaferro V, Regalia E, Doci R, et al. Liver transplantation for the treatment of small hepatocellular carcinomas in patients with cirrhosis. N Engl J Med. 1996;334(11):693-9.

9. Decaens T, Roudot-Thoraval F, Hadni-Bresson S, et al. Impact of UCSF criteria according to pre- and post-OLT tumor features: analysis of 479 patients listed for HCC with a short waiting time. Liver Transpl. 2006;12(12):1761-9.

10. Llovet JM, Burroughs A, Bruix J. Hepatocellular carcinoma. Lancet. 2003;362(9399):1907-17.

11.• Goldberg D, French B, Abt P, Feng S, Cameron AM. Increasing disparity in waitlist mortality rates with increased model for endstage liver disease scores for candidates with hepatocellular carcinoma versus candidates without hepatocellular carcinoma. Liver Transpl. 2012;18(4):434-43. This study explores and evaluates the waitlist mortality of patients with HCC MELD exceptions in comparison with waitlist candidates without exceptions, but with comparable MELD scores.
12. Massie AB, Caffo B, Gentry SE, et al. MELD Exceptions and Rates of Waiting List Outcomes. Am J Transplant. 2011.

13. Washburn K, Edwards E, Harper A, Freeman R. Hepatocellular carcinoma patients are advantaged in the current liver transplant allocation system. Am J Transplant. 2010;10(7):1643-8.

14. Bittermann T, Niu B, Hoteit MA, Goldberg D. Waitlist priority for hepatocellular carcinoma beyond milan criteria: a potentially appropriate decision without a structured approach. Am J Transplant. 2014;14(1):79-87.

15. Mehta N, Dodge JL, Goel A, Roberts JP, Hirose R, Yao FY. Identification of liver transplant candidates with hepatocellular carcinoma and a very low dropout risk: implications for the current organ allocation policy. Liver Transpl. 2013;19(12):1343-53.

16. OPTN/UNOS Policy and Bylaw Proposals Distributed for Public Comment. http://optn.transplant.hrsa.gov/policiesAndBylaws/ publicComment/proposals.asp. Accessed March 14, 2014.

17. Cucchetti A, Cescon M, Bigonzi E, et al. Priority of candidates with hepatocellular carcinoma awaiting liver transplantation can be reduced after successful bridge therapy. Liver Transpl. 2011;17(11): 1344-54.

18. Guiteau JJ, Cotton RT, Washburn WK, et al. An early regional experience with expansion of Milan Criteria for liver transplant recipients. Am J Transplant. 2010;10(9):2092-8.

19. Yao FY. Liver transplantation for hepatocellular carcinoma: beyond the Milan criteria. Am J Transplant. 2008;8(10):1982-9.

20. Goldberg DS, Krok K, Batra S, Trotter JF, Kawut SM, Fallon MB. Impact of the Hepatopulmonary Syndrome MELD Exception Policy on Outcomes of Patients After Liver Transplantation: An Analysis of the UNOS Database. Gastroenterology.2014.

21. Bittermann T, Makar G, Goldberg D. Exception point applications for 15 points: an unintended consequence of the share 15 policy. Liver Transpl. 2012;18(11):1302-9.

22. A H. MELD Exception Requests, May 1, 2012-April 30, 2013.

23. Biggins SW, Kim WR, Terrault NA, et al. Evidence-based incorporation of serum sodium concentration into MELD. Gastroenterology. 2006;130(6):1652-60.

24. Kim WR, Biggins SW, Kremers WK, et al. Hyponatremia and mortality among patients on the liver-transplant waiting list. $\mathrm{N}$ Engl J Med. 2008;359(10):1018-26.

25. Goldberg D, French B, Thomasson A, Reddy KR, Halpern SD. Waitlist survival of patients with primary sclerosing cholangitis in the model for end-stage liver disease era. Liver Transpl. 2011;17(11):1355-63.

26. Goldberg DSCA, Martinez-Camacho A, Forman L, Fortune B, Reddy KR. Risk of waitlist mortality in patients with primary sclerosing cholangitis and bacterial cholangitis. 2012.

27. Goldberg D, Bittermann T, Makar G. Lack of Standardization in Exception Points for Patients with Primary Sclerosing Cholangitis and Bacterial Cholangitis. Am J Transplant. 2012.

28. Yeh H, Smoot E, Schoenfeld DA, Markmann JF. Geographic inequity in access to livers for transplantation. Transplantation. 2011;91(4):479-86. 